\title{
Structural Studies of the Self-Assemblies Created with Dipyrrins
}

\author{
Ji-Young Shin, Brian O. Patrick, Seung Bae Son, ${ }^{\dagger}$ Jae Ryang Hahn, ${ }^{\dagger}$ and David Dolphin* \\ Department of Chemistry, University of British Columbia, 2036 Main Mall, Vancouver B.C. V6T 1Z1, Canada \\ ${ }^{*}$ E-mail: david.dolphin@ubc.ca \\ 'Department of Chemistry, Chonbuk National University and Research Institute of Physics and Chemistry, \\ Jeonju 561-756, Korea \\ Received December 15, 2009, Accepted January 23, 2010
}

\begin{abstract}
Three-dimensional superstructures of unique self-assemblies generated by exploring the conformational flexibility of various dipyrromethenes through creation of hydrogen-bonds with metal-halide anions are reported and the conformational diversity is thoroughly described in the solid and solution states by X-ray diffraction analysis and variable temperature NMR spectroscopy. The tetrahedral or octahedral structures of their precursors, various metal-dipyrromethene complexes, are also reported, based on the crystallographic data. STM images of the self-assemblies observed on graphite surfaces present interesting arrangements and appear as tubular bunches.
\end{abstract}

Key Words: Dipyrromethene, Metal complex, Hydrogen-bond, STM

\section{Introduction}

The chemistry of inter/intramolecular interactions that result in the self-assembly of supramolecules is attracting considerable interest in many diverse areas of chemistry, physics, engineering and crystal design. ${ }^{1-11}$ Self-assemblies of supramolecules may be formed via weak inter/intramolecular interactions such as metal-organic, hydrogen-bonding, electrostatic and hydrophobic interaction and van der Waals interactions. The fact that metal-ligand interactions $(20-80 \mathrm{kcal} / \mathrm{mole})$ are smaller than covalent bond strengths, which are typically $100 \mathrm{kcal} / \mathrm{mole}$, allows for many different ligands to be introduced into self-assemblies. Over the past decade, porphyrins and related polypyrrolyl analogues have been examined as interesting building blocks for self-assemblies due to their versatile metal-coordination capacities. ${ }^{12-16}$ Alternatively, hydrogen-bonding and electrostatic and hydrophobic interactions which are typically less than $10 \mathrm{kcal} / \mathrm{mole}$ in general, are also important factors in the formation of self-assemblies. Unique 3-dimensional self-assem- bled structures generated through inter/intramolecular hydrogen-bonding interactions can be expected when using polypyrrolyl analogues having amino-type and imino-type nitrogens (or other strong hydrogen-bond-acceptors such as oxo-subs-

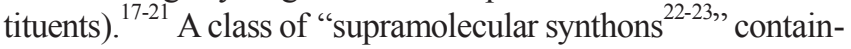
ing both hydrogen-bonding donors and acceptors can lead to the creation of diverse types of new self-assemblies through hydrogen-bonding networks, which control the packing of the "molecular tectons". ${ }^{24-26}$
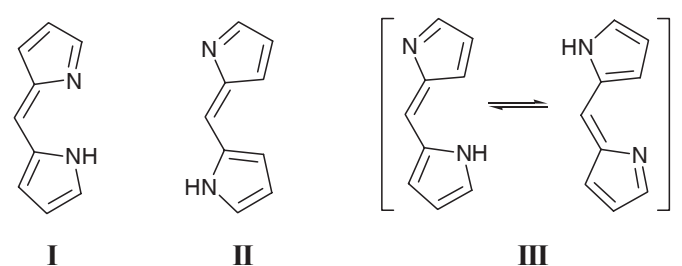

Figure 1. Three possible configurations of dipyrromethene.
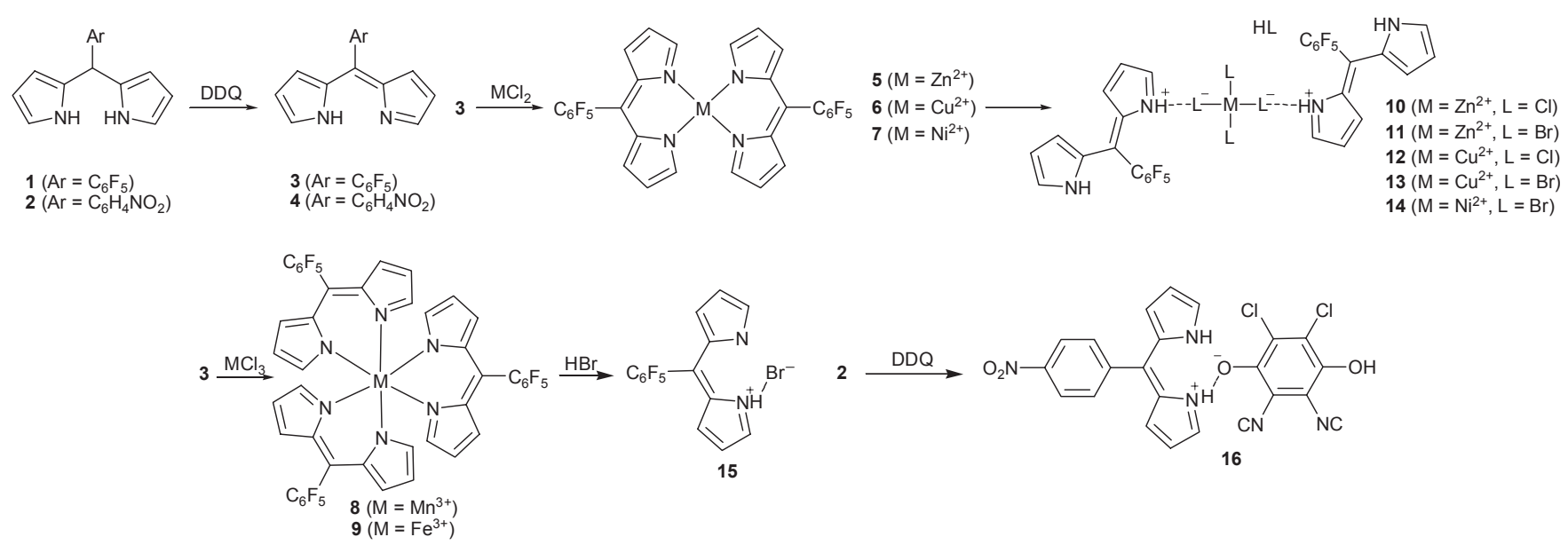

2

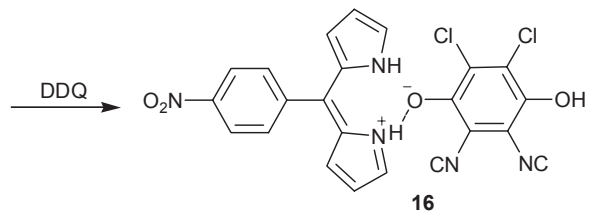

Scheme 1. Synthetic process 


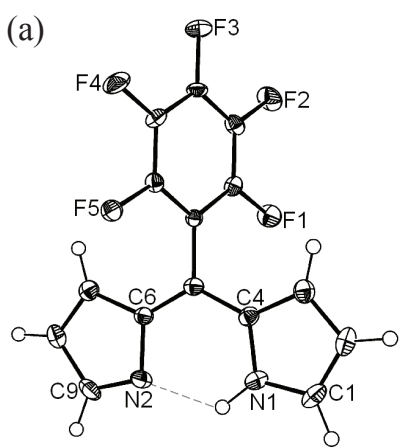

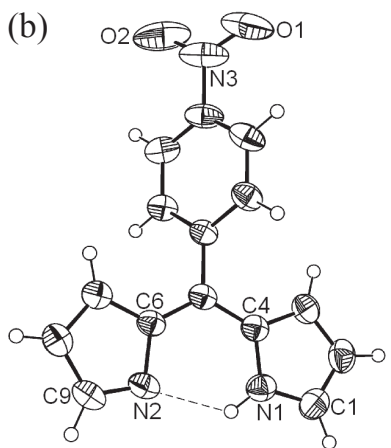

Figure 2. Crystal structures of dipyrromethenes (a) 3 and (b) $4 .{ }^{29}$

(a)

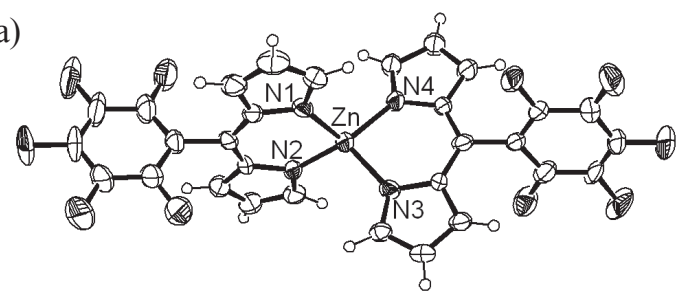

(b)

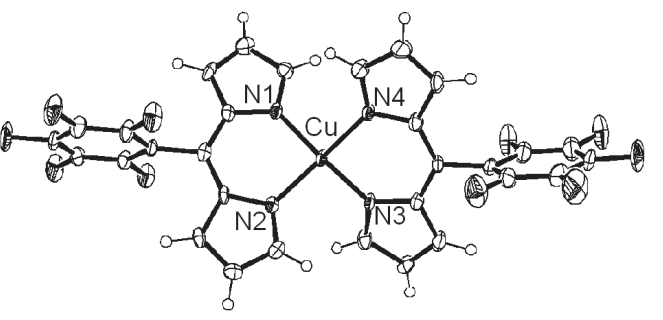

Figure 3. Crystal structures of (a) $\mathrm{Zn}^{2+}$ dipyrromethene 5 and (b) $\mathrm{Cu}^{2+}$ dipyrromethene 6 .

Dipyrrins exhibit rotations of the pyrrolyl groups around the central methene-bridge and three different conformations are expected (types I, II and III in figure 1). ${ }^{27}$ Recently, we reported several unique self-assemblies in which protonated dipyrrins were crystallized with metal-halide anions. ${ }^{28}$ When dipyrrins form crystals of various self-assemblies with secondary components, the conformational dynamics of the dipyrrin components play a key role. Consequently, the electronic features of the dipyrrin components affect these dynamics. Dipyrrins 3 and 4 (Scheme 1), which contain electron-withdrawing groups on the meso-aryls and are unsubstituted in the $\alpha$-positions, are chemically stable and conformationally mobile. In this respect, 3 and $\mathbf{4}$ have been suggested as good candidates for incorporation into further structures.

Free-base dipyrrins and metal-dipyrrin complexes exist mostly in the type I form via intramolecular hydrogen-bonding between imino- and amino-N atoms and ligand-metal chelation (see Figures 2-4). Dicationic metal complexes of $\mathbf{3}, \mathrm{ZnD}_{2} \mathbf{5}$ and $\mathrm{CuD}_{2}$ 6 (having a 1:2 binding ratio for metal and dipyrrins to form tetrahedral complexes) and tricationic metal complexes of $\mathbf{3}$, $\mathrm{MnD}_{3} 8$ and $\mathrm{FeD}_{3} 9$ (having 1:3 binding as a result of octahedral coordination) have been prepared. All of their crystal structures reveal type I conformation of the dipyrrin moiety.

When the metal complexes (5-7) were treated with hydrogen

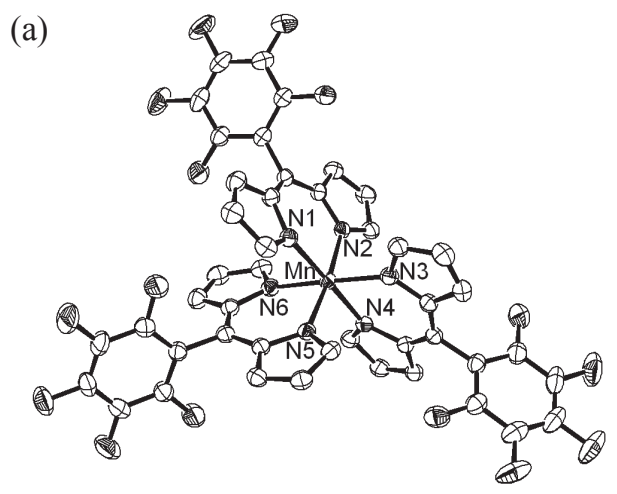

(b)

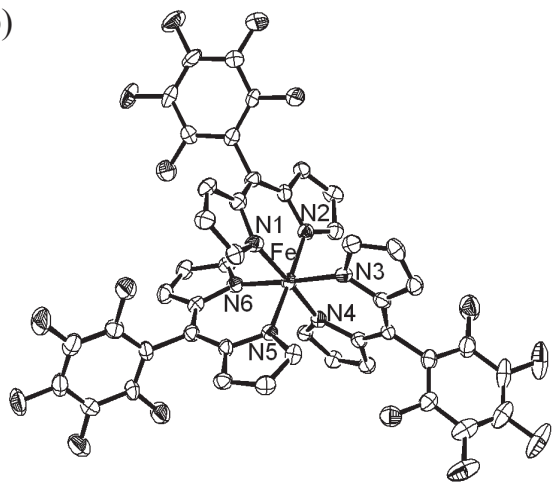

Figure 4. Crystal structures of (a) $\mathrm{Mn}^{3+}$ dipyrromethene 8 and (b) $\mathrm{Fe}^{3+}$ dipyrromethene 9 ; H-atoms have been omitted for clarity.

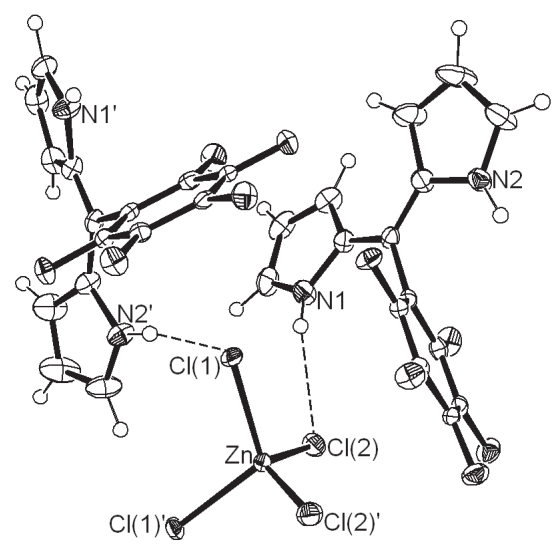

Figure 5. ORTEP structure ${ }^{28}$ of $\mathbf{1 0}$.

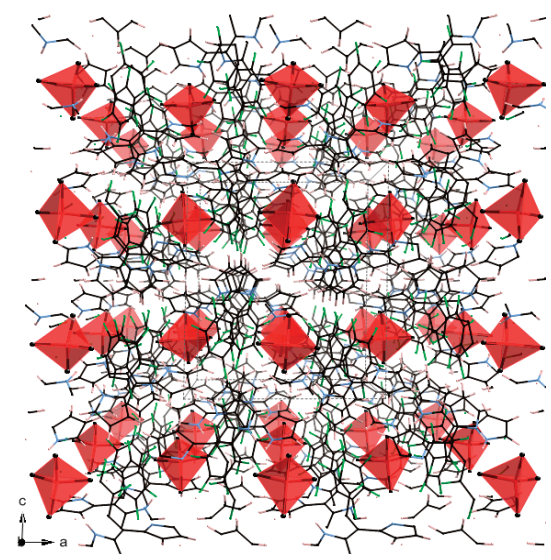

Figure 6. 3D-packing diagram of $\mathbf{1 0}$. 

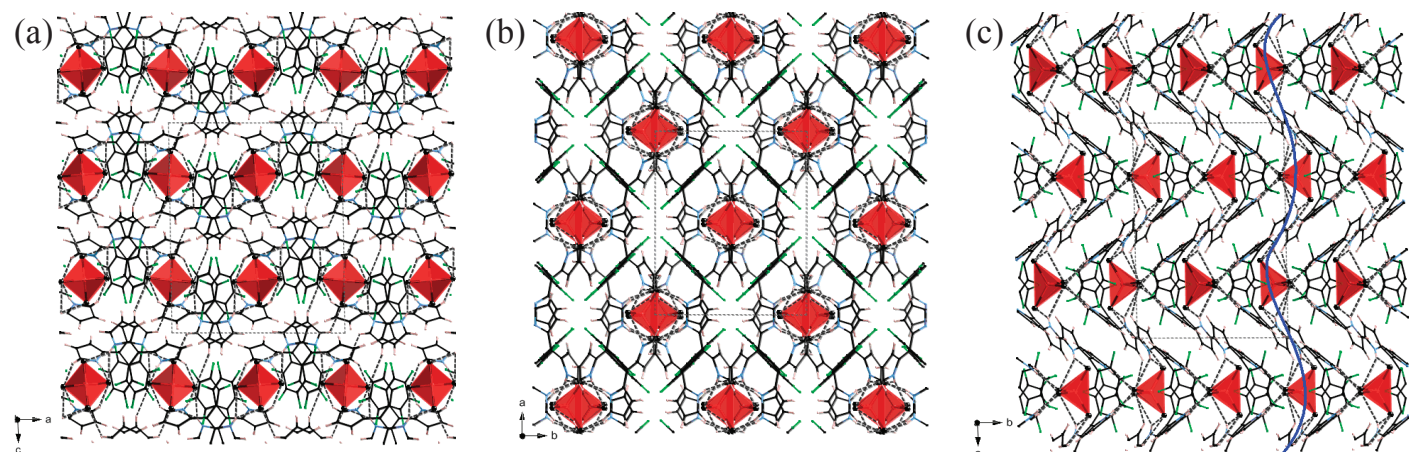

Figure 7. Packing diagrams of $\mathbf{1 0}$ through each side; (a) ac, (b) ab, and (c) bc planes. Hydrogen-bonds have been displayed as dotted lines.

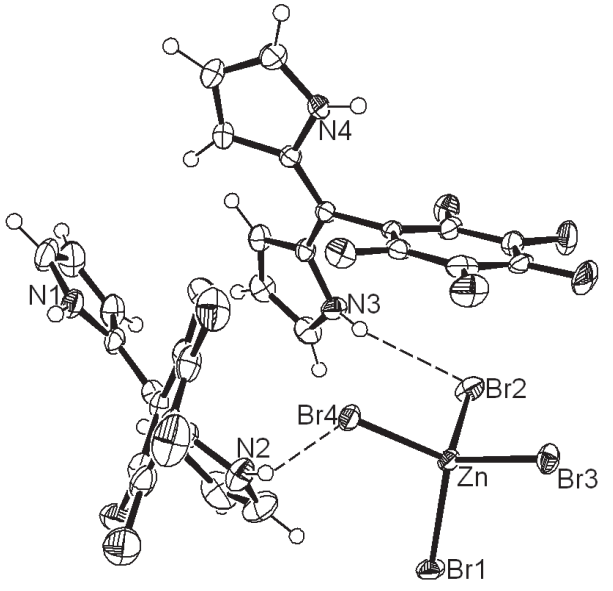

Figure 8. ORTEP structure ${ }^{28}$ of $\mathbf{1 1}$

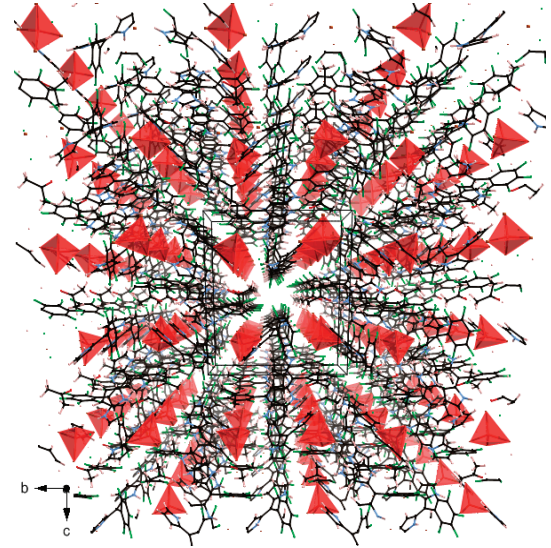

Figure 9. 3D packing diagram of 11 .
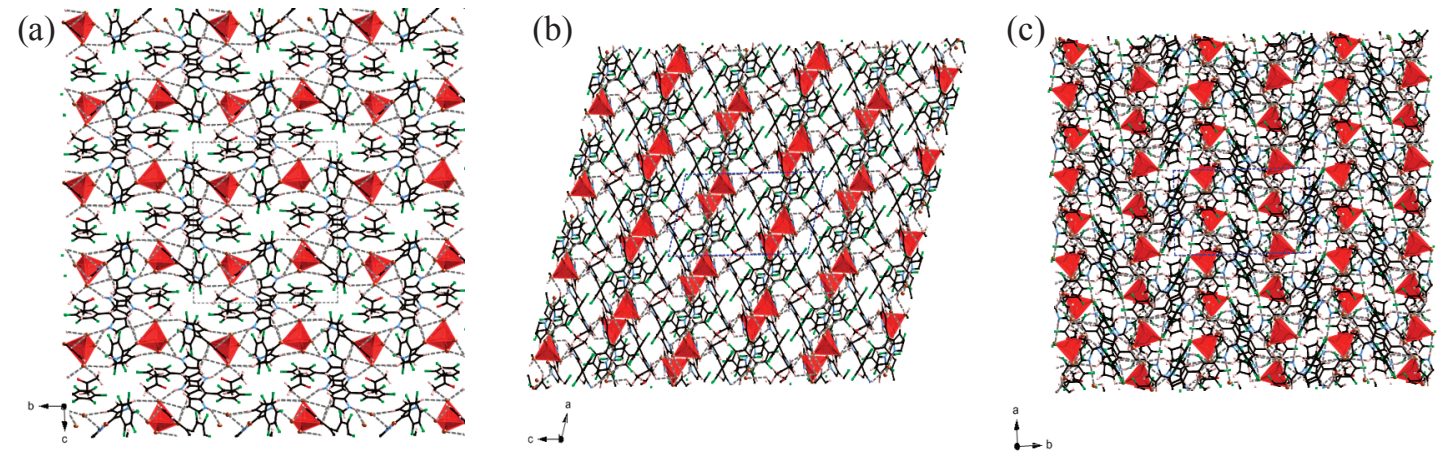

Figure 10. Packing diagrams of $\mathbf{1 1}$ through each side; (a) bc, (b) ac, and (c) ab planes. Hydrogen-bonds have been displayed as dotted lines.

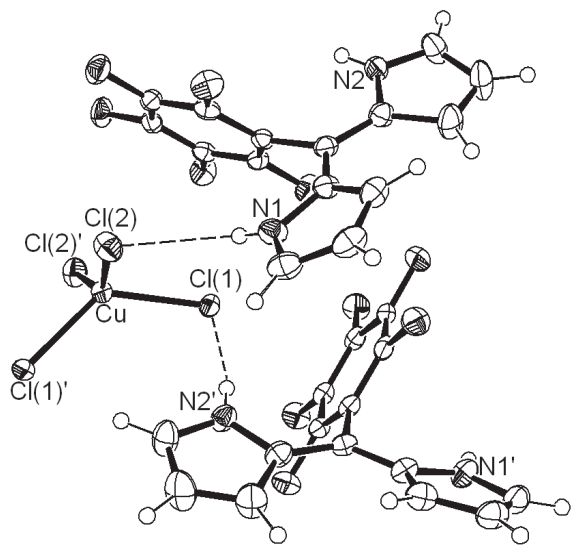

halides $(\mathrm{HF}, \mathrm{HCl}$, and $\mathrm{HBr}$ ), new self-assemblies of protonated dipyrrins were obtained: $\mathrm{ZnCl}_{4}(\mathrm{HD})_{2} \mathbf{1 0}, \mathrm{ZnBr}_{4}(\mathrm{HD})_{2} \mathbf{1 1}, \mathrm{CuCl}_{4}$ $(\mathrm{HD})_{2} 12, \mathrm{CuBr}_{4}(\mathrm{HD})_{2} 13, \mathrm{NiBr}_{4}(\mathrm{HD})_{2} 14$ and $\mathrm{HDBr}$ 15. The structures of 10-12 and 15 were also determined by X-ray diffraction crystallography and exhibit film-like (Figures 7, 10, and 14) and ribbon-like (Figure 16) self-assemblies in the solid state. These self-assemblies employ the type II conformations of the dipyrrin units. Self-assemblies taking the type III conformation in the solid state remained elusive until we solved the crystal structure for $16 .{ }^{29}$ Figure 17 shows the quinolate units form hydrogen-bonds with two different protonated dipyrrins on each oxygen, and the dipyrrin units display the type III conformation. The structural details of self-assemblies utilizing all three dipyrrin conformations are reported in this paper. Furthermore, uni-

Figure 11. ORTEP structure of $\mathbf{1 2}$. 

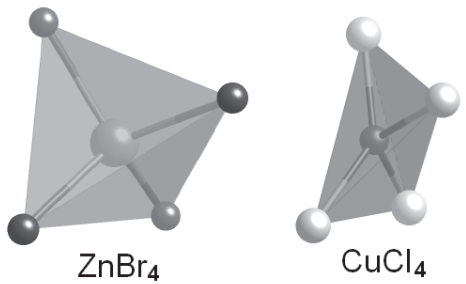

Figure 12. Tetrahedral structural comparison between $\mathrm{ZnBr}_{4}$ and $\mathrm{CuCl}_{4}$ in the corresponding crystals.

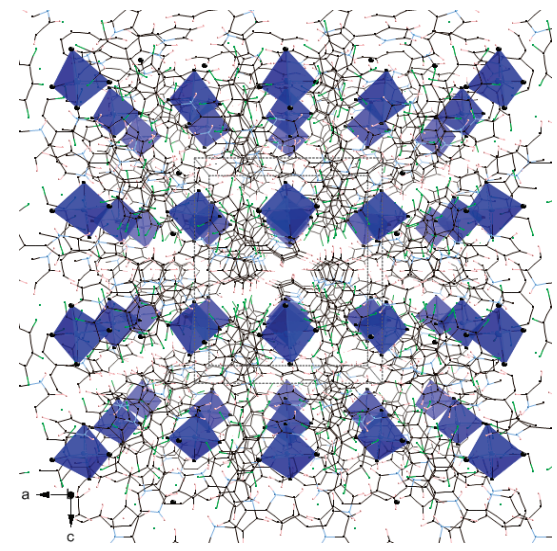

Figure 13. 3D packing diagram of $\mathbf{1 2}$.
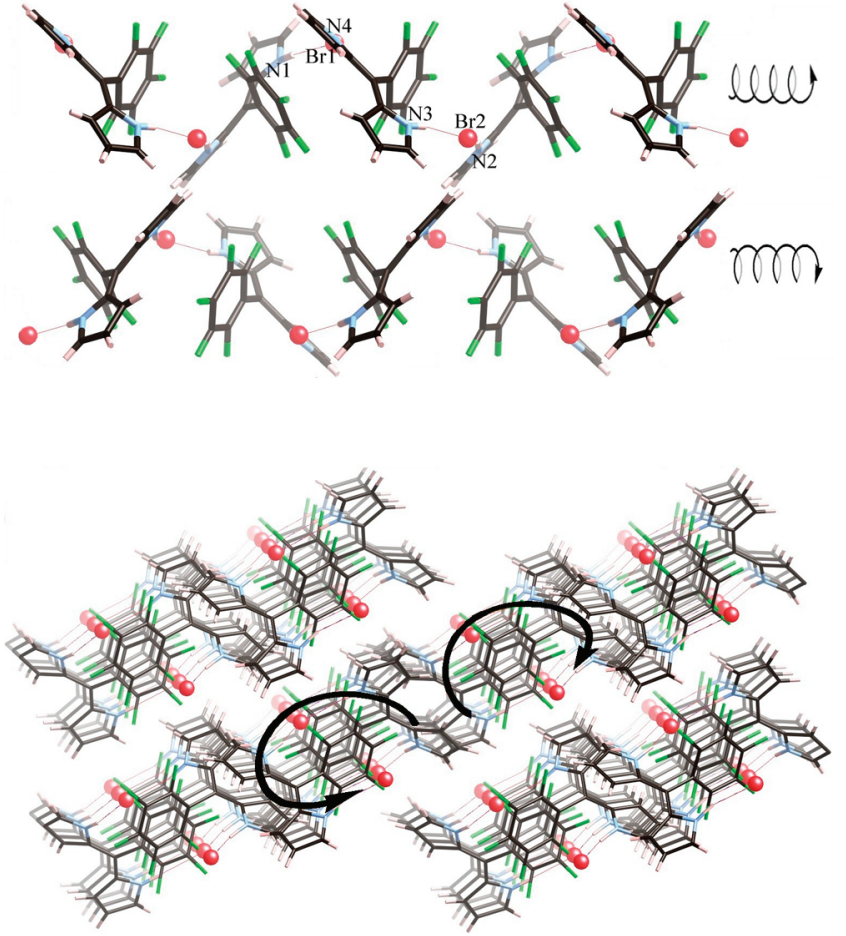

Figure 16. 3D packing diagrams of $\mathbf{1 5 .}$ (a)

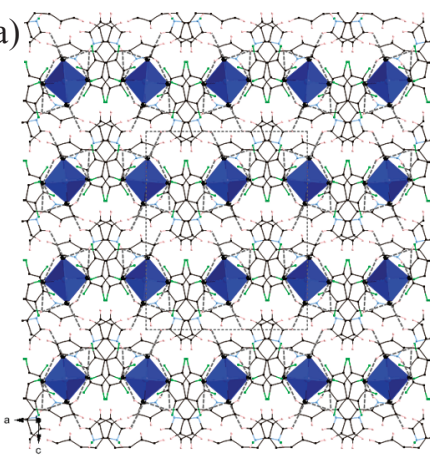

(b)

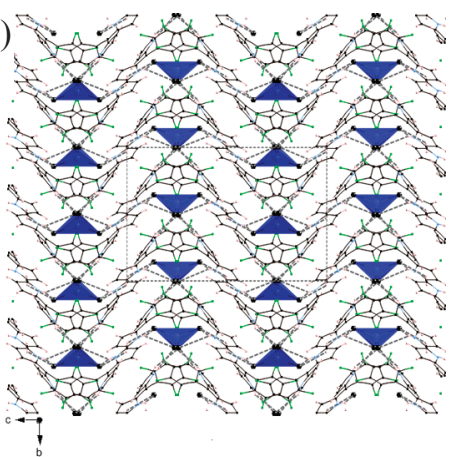

(c)

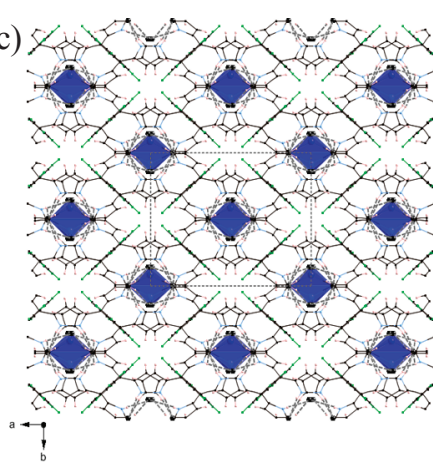

Figure 14. Packing diagrams of 12 through each side; (a) ac, (b) bc, and (c) ab planes. Hydrogen-bonds have been displayed as dotted lines.

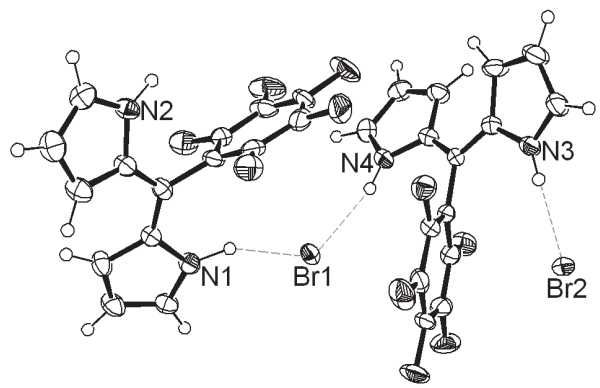

Figure 15. ORTEP structure ${ }^{28}$ of $\mathbf{1 5 .}$

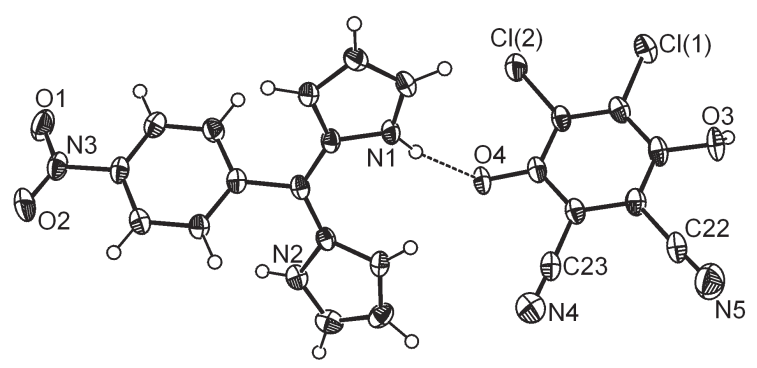

Figure 17. ORTEP structure ${ }^{29}$ of $\mathbf{1 6 .}$ 
que "tubular-bunched" arrangements (through long-range hydrogen-bonding) of these self-assemblies were observed with scanning tunneling microscopy (STM).

\section{Experimental}

General. All chemicals were purchased from commercial suppliers and used without further purification. Column chromatography of all products was carried out using silica gel (particle size: $0.040-0.063 \mathrm{~mm}, 230$ - 400 mesh). ${ }^{1} \mathrm{H}$ and ${ }^{13} \mathrm{C}$ NMR spectra were recorded with Bruker Avance 300 and 400 NMR spectrometers in $\mathrm{CD}_{2} \mathrm{Cl}_{2}, \mathrm{CDCl}_{3}, d_{6}$-acetone, or $d_{6}$-DMSO. Mass spectra were determined on EI, ESI or MALDI-TOF mass spectrometers. Optical spectra were recorded with a Cary 5000 UVvis spectrophotometer using a $1 \mathrm{~cm}$ cell. FTIR spectra were recorded using their $\mathrm{KBr}$ pellets.

Preparation of compounds 1-4. Compounds 1-2 were prepared following the literature method. ${ }^{30}$ By treatment of the $\mathrm{CH}_{2} \mathrm{Cl}_{2}$ solutions of $\mathbf{1}$ and $\mathbf{2}$ with DDQ, compounds 3-4 were prepared quantitatively. Spectral data of 1: (67\% yield, white powder) $\mathrm{mp} 127 \sim 128{ }^{\circ} \mathrm{C}$; ${ }^{1} \mathrm{H}$ NMR at $\mathrm{rt}\left(\mathrm{CDCl}_{3}, 300 \mathrm{MHz}\right) \delta$ 8.08 (bs, $2 \mathrm{H}), 6.71$ (m, $J=1.5,2 \mathrm{H}), 6.15$ (dd, $J=2.8$, dd, $J=6.0$, $2 \mathrm{H}), 6.01$ (s, 2H). Spectral data of 2: (42\% yield, light yellow powder) $\mathrm{mp} 167{ }^{\circ} \mathrm{C} ;{ }^{1} \mathrm{H}$ NMR at $\mathrm{rt}\left(\mathrm{CDCl}_{3}, 300 \mathrm{MHz}\right) \delta 8.15$ $(\mathrm{d}, J=8.8,2 \mathrm{H}), 7.95$ (bs, 2H), 7.35 (d, $J=8.4,2 \mathrm{H}) ; \mathrm{m} / z$ EI 145 $\left(75 \%,\left[\mathrm{M}-\mathrm{C}_{6} \mathrm{H}_{4} \mathrm{NO}_{2}\right]^{+}\right), 201\left(27 \%,\left[\mathrm{M}-\mathrm{C}_{4} \mathrm{H}_{4} \mathrm{~N}\right]^{+}\right), 267(100 \%$, $\left.\mathrm{M}^{+}\right)$; Elemental analysis (\%) Calcd. 15.94(N), 68.31(C), 4.42(H) found 15.84(N), 67.92(C), 4.18(H). Spectral data of 3: (reddish powder) ${ }^{1} \mathrm{HNMR}$ at $\mathrm{rt}\left(\mathrm{CDCl}_{3}, 300 \mathrm{MHz}\right) \delta 12.7$ (bs, $\left.1 \mathrm{H}\right), 7.71$ (s, 2H), $6.52(\mathrm{~d}, J=4.2,2 \mathrm{H}), 6.46(\mathrm{dd}, J=4.3$, dd, $J=1.4,2 \mathrm{H})$, ${ }^{19} \mathrm{~F} \mathrm{NMR}\left(\mathrm{CD}_{2} \mathrm{Cl}_{2}, 282.4 \mathrm{MHz}\right) \delta-139.96$ (dd, $J=19.8$, dd, $J=$ $5.6,2 \mathrm{~F}, \mathrm{o}-\mathrm{F}),-153.60$ (m, 1F, p-F), -162.14 (dt, $J=19.8, \mathrm{dt}, J=$ 5.6, $2 \mathrm{~F}, \mathrm{~m}-\mathrm{F}$ ). Spectral data of 4 : (reddish powder) $\mathrm{mp} 120{ }^{\circ} \mathrm{C}$; $\lambda_{\max }\left(\mathrm{CH}_{2} \mathrm{Cl}_{2}\right) / \mathrm{nm} 438.0 ;{ }^{1} \mathrm{H}$ NMR at $\mathrm{rt}\left(\mathrm{CD}_{2} \mathrm{Cl}_{2}, 300 \mathrm{MHz}\right) \delta$ 12.95 (bs, $1 \mathrm{H}), 8.31$ (d, $J=8.8,2 \mathrm{H}), 6.49$ (dd, $J=4.2$, dd, $J=1.0$, $2 \mathrm{H}) ;{ }^{13} \mathrm{CNMR}\left(\mathrm{CD}_{2} \mathrm{Cl}_{2}, 75.5 \mathrm{MHz}\right) \delta 148.8,145.1,144.3,140.9$, 139.3, 132.2, 128.7, 123.4, 118.9; HR ESI calcd. $266.0930 \mathrm{~m} / \mathrm{z}$ (for $\mathrm{C}_{15} \mathrm{H}_{12} \mathrm{~N}_{3} \mathrm{O}_{2}$ ), found $266.0933 \mathrm{~m} / \mathrm{z}$.

Preparation of metal complexes 5-9. $\mathrm{A} \mathrm{CH}_{3} \mathrm{CN}(100 \mathrm{~mL})$ solution of meso-pentafluorophenyl dipyrromethane $(350 \mathrm{mg}$, $1.12 \mathrm{mmole}$ ) was placed in a $250 \mathrm{~mL}$ round bottom flask. The resulting solution was oxidized for $40 \mathrm{~min}$ by addition of DDQ (255 mg, $1.12 \mathrm{mmole}$ ), and then quenched with $0.5 \mathrm{~mL}$ of triethylamine. $80 \mathrm{~mL}$ of $\mathrm{MeOH}$ solution of $\mathrm{ZnCl}_{2}(84 \mathrm{mg}, 618$ mmole) was added into the solution, and the reaction was monitored by TLC. After the reaction was completed, the solvent was removed on a rotary evaporator. The residue was purified by column chromatography on silica gel with $\mathrm{CH}_{2} \mathrm{Cl}_{2}$ and recrystallized from $\mathrm{CH}_{2} \mathrm{Cl}_{2} / \mathrm{MeOH}$ to obtain compound $\mathbf{5}$ in $c a$. 48\% yield. Complexes 6-9 were prepared from corresponding metal salts following the same method ( $c a .13 \sim 20 \%$ yields). Spectral data of $\mathrm{Zn}^{2+}$ complex 5: $\mathrm{mp}>230{ }^{\circ} \mathrm{C} ;{ }^{1} \mathrm{H}$ NMR at $\mathrm{rt}$ $\left(\mathrm{CD}_{2} \mathrm{Cl}_{2}, 300 \mathrm{MHz}\right) \delta 7.59(\mathrm{~s}, 4 \mathrm{H}), 6.70(\mathrm{~d}, J=4.2,4 \mathrm{H}), 6.49$ (dd, $J=4.2, \mathrm{dd}, J=1.3,4 \mathrm{H}) ;{ }^{19} \mathrm{~F} \mathrm{NMR}$ at $\mathrm{rt}\left(\mathrm{CD}_{2} \mathrm{Cl}_{2}, 282.4 \mathrm{MHz}\right)$ $\delta-63.59(\mathrm{dd}, J=22.8, \mathrm{dd}, J=6.6,4 \mathrm{~F}),-77.21(\mathrm{t}, J=21.9,2 \mathrm{~F})$, -85.45 (tt, $J=22.2, \mathrm{tt}, J=6.3,4 \mathrm{~F}) ;{ }^{13} \mathrm{C} \mathrm{NMR}$ at $\mathrm{rt}\left(\mathrm{CD}_{2} \mathrm{Cl}_{2}, 75.5\right.$ $\mathrm{MHz}) \delta 152.1,140.0,131.6,119.1$ (peaks of meso-aryl Cs are broaden due to C-F couplings); $v_{\max }(\mathrm{KBr}) / \mathrm{cm}^{-1} 2957.8 \mathrm{~s}, 2919.8 \mathrm{~s}$,
$2839.1 \mathrm{~s}, 1734.1 \mathrm{w}, 1557.7 \mathrm{~m}, 1522.3 \mathrm{~m}, 1458.5 \mathrm{~s}, 1378.0 \mathrm{~s}$, $1338.0 \mathrm{w}, 1243.5 \mathrm{w}, 1165.7 \mathrm{w}, 1030.6 \mathrm{~m}, 992.9 \mathrm{~m}, 842.0 \mathrm{w}$, $750.8 \mathrm{w}$; UV-vis $\left(\mathrm{CH}_{2} \mathrm{Cl}_{2}\right) \lambda_{\max } / \mathrm{nm}(\log \varepsilon) 304$ (4.1), 370 (4.2), 496 (5.2); LSIMS $682.2 \mathrm{~m} / \mathrm{z}\left(\mathrm{M}^{+}\right)$; Elemental analysis (\%) Calcd. 8.19(N), 52.69(C), 1.77(H) found 8.31(N), 53.00(C), 1.85(H). Spectral data of $\mathrm{Cu}^{2+}$ complex 6: $\mathrm{mp}>230{ }^{\circ} \mathrm{C}$; $v_{\max }$ $(\mathrm{KBr}) / \mathrm{cm}^{-1} 2957.6 \mathrm{~s}, 2920.9 \mathrm{~s}, 2839.5 \mathrm{~s}, 1733.8 \mathrm{w}, 1560.3 \mathrm{w}$, $1458.4 \mathrm{~s}, 1377.8 \mathrm{~s}, 1165.8 \mathrm{~m}, 998.0 \mathrm{w}, 973.4 \mathrm{w}, 814.2 \mathrm{w} ; 8.10$ (bs, $2 \mathrm{H})$, LSIMS $682.0 \mathrm{~m} / z\left(\mathrm{M}^{+}\right) ; v_{\max }\left(\mathrm{CH}_{2} \mathrm{Cl}_{2}, \log \varepsilon\right) / \mathrm{nm} 380$ (4.2), 476 (4.8), 512 (4.5). Spectral data of $\mathrm{Ni}^{2+}$ complex 7: mp > $230{ }^{\circ} \mathrm{C} ; v_{\max }(\mathrm{KBr}) / \mathrm{cm}^{-1} 2958.8 \mathrm{~s}, 2920.9 \mathrm{~s}, 2839.2 \mathrm{~s}, 1734.3 \mathrm{w}$, $1560.7 \mathrm{w}, 1458.5 \mathrm{~s}, 1378.0 \mathrm{~s}, 1165.7 \mathrm{w}, 996.9 \mathrm{w}, 841.7 \mathrm{w}, 753.5 \mathrm{w}$; LSIMS $677.04 \mathrm{~m} / \mathrm{z}\left(\mathrm{M}^{+}\right)$. Spectral data of $\mathrm{Mn}^{3+}$ complex 8: ${ }^{1} \mathrm{H}$ $\mathrm{NMR}$ at $\mathrm{rt}\left(\mathrm{CD}_{2} \mathrm{Cl}_{2}, 300 \mathrm{MHz}\right) \delta 19.31(\mathrm{bs}, 6 \mathrm{H}), 6.45(\mathrm{~d}, J=$ $18.4,6 \mathrm{H}),-31.04$ (bs, 6H). Spectral data of $\mathrm{Fe}^{3+}$ complex 9: $\mathrm{mp}>230{ }^{\circ} \mathrm{C} ;{ }^{1} \mathrm{H}$ NMR at $\mathrm{rt}\left(d_{6}\right.$-acetone, $\left.300 \mathrm{MHz}\right) \delta-6.20(\mathrm{~s}$, $6 \mathrm{H}),-7.22(\mathrm{~s}, 6 \mathrm{H}),-28.78(\mathrm{~s}, 6 \mathrm{H}) ;{ }^{19} \mathrm{~F}$ NMR at rt $\left(d_{6}\right.$-acetone, $282.4 \mathrm{MHz}) \delta-62.01(\mathrm{dd}, J=23.7, \mathrm{dd}, J=6.3,2 \mathrm{H}),-78.65(\mathrm{t}$, $J=21.9,1 \mathrm{H}),-86.23(\mathrm{t}, J=22.2,2 \mathrm{H})$; ESIMS $984.1 \mathrm{~m} / \mathrm{z}\left(\mathrm{M}^{+}\right)$; Elemental analysis (\%) Calcd. 8.55(N), 54.96(C), 1.85(H) found 8.61(N), 54.84(C), 1.71(H); $\lambda_{\max }\left(\mathrm{CH}_{2} \mathrm{Cl}_{2}\right) / \mathrm{nm} \mathrm{450,} 500$.

Preparation of self-assemblies 10-16. $20 \mathrm{~mL}$ of $\mathrm{CH}_{2} \mathrm{Cl}_{2}$ solution of $5(90.2 \mathrm{mg}, 132 \mu \mathrm{mole})$ was placed in a $50 \mathrm{~mL}$ round bottom flask, and degassed under nitrogen. $110 \mu \mathrm{L}$ of $30 \% \mathrm{HBr}$ solution in acetic acid was added by syringe and the resulting solution was stirred for $6 \mathrm{hr}$. Precipitates, which appeared during the reaction, were filtered and washed with $\mathrm{CH}_{2} \mathrm{Cl}_{2}$, hexane, and water. The title compound 11 was obtained in $c a$. $60 \%$ yield. Compound 10 was obtained from $35 \% \mathrm{HCl}$ solution by the same method, and compound 15 was obtained by treating 9 with excess HBr. Self-assemblies 10 and 12-14 were also prepared by the same method using corresponding metal complexes and hydrogen halides ( $c a .50 \sim 85 \%$ ); Solubility differences between the metal complexes and the self-assemblies were significant. While the class of metal complexes showed reasonable solubility in $\mathrm{CH}_{2} \mathrm{Cl}_{2}$, the self-assemblies showed poor solubility in the same solvent. Also, the self-assemblies containing paramagnetic metals resonated broad signals in the regular aromatic region in their NMR spectra, whereas the metal complexes had no such resonance. The dipyrrin resonances are likely less affected by the metal in the self-assemblies by the disruption of direct metal coodination and enhancement of secondary hydrogen-bond formation. Consequently, the mass spectra of the self-assembies showed various patterns of coordination between protonated dipyrrin and metal halide synthons due to the relatively weaker interactions of hydrogen-bonds. Spectral data for $\mathrm{ZnCl}_{4}(\mathrm{HD})_{2}$ 10: $\mathrm{mp} 180 \sim 182{ }^{\circ} \mathrm{C} ;{ }^{1} \mathrm{HNMR}$ at $360 \mathrm{~K}\left(d_{6}\right.$ - DMSO, $300 \mathrm{MHz}) \delta 8.08(\mathrm{~s}, 2 \mathrm{H}), 7.36(\mathrm{bs}, 2 \mathrm{H}), 6.75(\mathrm{~s}, 2 \mathrm{H}) ;{ }^{19} \mathrm{~F} \mathrm{NMR}$ at $210 \mathrm{~K}\left(d_{6}\right.$-acetone, $\left.282.4 \mathrm{MHz}\right), \delta-61.78(\mathrm{~d}, J=23.7,2 \mathrm{~F}$, o-F of first component), -62.86 (d, $J=19.8,2 \mathrm{~F}, \mathrm{o}-\mathrm{F}$ of second component), -65.55 (m, 2F, o-F of third component), -75.62 (m, 1F, p-F of first component), $-74.8(\mathrm{~m}, 1 \mathrm{~F}, \mathrm{p}-\mathrm{F}$ of second component), $-77.6(\mathrm{~m}, 1 \mathrm{~F}, \mathrm{p}-\mathrm{F}$ of third component), -85.22 ( $\mathrm{t}$, $J=19.5, \mathrm{~m}-\mathrm{F}$ of first component), -85.53 (t, $J=22,2 \mathrm{~F}, \mathrm{~m}-\mathrm{F}$ of second component), -85.92 ( $\mathrm{m}, 2 \mathrm{~F}, \mathrm{~m}-\mathrm{F}$ of third component); $v_{\max }$ $(\mathrm{KBr}) / \mathrm{cm}^{-1} 2959 \mathrm{~s}, 2920 \mathrm{~s}, 2839 \mathrm{~m}, 1735 \mathrm{w}, 1504 \mathrm{w}, 1456 \mathrm{~s}, 1377 \mathrm{~s}$, $1332 \mathrm{~m} \mathrm{1141m,} \mathrm{1046s,} \mathrm{997m,} \mathrm{972m,} \mathrm{868w,} \mathrm{836w,} \mathrm{773w;} \mathrm{TOF}$ $\mathrm{ES}+792.9 \mathrm{~m} / \mathrm{z}\left([\mathrm{M}-\mathrm{Cl}]^{+}\right)$; Elemental analysis (\%) Calcd. 6.75 
$(\mathrm{N}), 43.43(\mathrm{C}), 1.94(\mathrm{H})$, found 6.69(N), 43.69(C), 1.89(H). Spectral data for $\mathrm{ZnBr}_{4}(\mathrm{HD})_{2} 11: \mathrm{mp} 220 \sim 226{ }^{\circ} \mathrm{C} ;{ }^{1} \mathrm{H} \mathrm{NMR}$ at $333 \mathrm{~K}$ $\left(d_{6}\right.$-DMSO, $\left.300 \mathrm{MHz}\right) \delta 8.17$ (s, 2H), 7.49 (br s, 2H), 6.83 (s, $2 \mathrm{H}) ;{ }^{19} \mathrm{~F}$ NMR at $330 \mathrm{~K}\left(d_{6}-\mathrm{DMSO}, 282.4 \mathrm{MHz}\right), \delta-63.98(\mathrm{bs}$, $2 \mathrm{~F}),-75.38$ (bs, 1F), $-85.07(\mathrm{~m}, 2 \mathrm{~F}) ; v_{\max }(\mathrm{KBr}) / \mathrm{cm}^{-1} 2955 \mathrm{~s}$, 2921s, 2839m, 1733w, 1561w, 1503w, 1457s, 1378s, 1332w, 1140w, 1047m, 998m, 973m, 839w; TOF ES+926.8 m/z ([M$\left.\mathrm{Br}]^{+}\right)$; Elemental analysis (\%) Calcd. 5.56(N), 35.74(C), 1.60 $(\mathrm{H}), 31.72(\mathrm{Br})$ found $5.34(\mathrm{~N}), 35.51(\mathrm{C}), 1.52(\mathrm{H}), 31.43(\mathrm{Br}) \lambda_{\max }$ $\left(\mathrm{CH}_{2} \mathrm{Cl}_{2}, \log \varepsilon\right) / \mathrm{nm} 478$ (4.6). Spectral data for $\mathrm{CuCl}_{4}(\mathrm{HD})_{2}$ 12: TOF ES+ $1030.1 \mathrm{~m} / z\left(\left[\mathrm{M}+\mathrm{H}_{2} \mathrm{CuBr}_{4}\right]^{+}\right.$; detected using the single crystals). Spectral data for $\mathrm{CuBr}_{4}(\mathrm{HD})_{2}$ 13: mp $187 \sim 189$ ${ }^{\circ} \mathrm{C} ;{ }^{1} \mathrm{H}$ NMR at $\mathrm{rt}\left(d_{6}\right.$-DMSO, $\left.400 \mathrm{MHz}\right) \delta 13.06$ (bs, 2H, NH), 8.31 (bs, 2H), 8.00 (bs, $2 \mathrm{H}), 6.96$ (bs, $2 \mathrm{H}) ; v_{\max }(\mathrm{KBr}) / \mathrm{cm}^{-1}$ $2958 \mathrm{~s}, 2921 \mathrm{~s}, 2839 \mathrm{~m}, 1734 \mathrm{w}, 1560 \mathrm{w}, 1458 \mathrm{~s}, 1378 \mathrm{~s}, 1166 \mathrm{~m}$, 998w, 973w, 841w; TOF ES+ $1021.3 \mathrm{~m} / z\left([\mathrm{M}+\mathrm{Na}]^{+}\right)$. Spectral data for $\mathrm{NiBr}_{4}(\mathrm{HD})_{2} \mathbf{1 4}: \mathrm{mp}>230{ }^{\circ} \mathrm{C} ;{ }^{1} \mathrm{H} \mathrm{NMR}$ at $\mathrm{rt}\left(d_{6}\right.$-DMSO, $400 \mathrm{MHz}$ ) $\delta 13.05$ (bs, 2H), 8.28 (bs, 2H), 8.10 (bs, 2H), 6.94 (bs, 2H); $v_{\max }(\mathrm{KBr}) / \mathrm{cm}^{-1} 2957 \mathrm{~s}, 2921 \mathrm{~s}, 2840 \mathrm{~m}, 1734 \mathrm{w}, 1459 \mathrm{~s}$, 1378s, 1166m, 998w, 974w, 841w; TOF ES+685.1 m/z ([M$\mathrm{D}]^{+}$). Spectral data for $\mathrm{HDBr} 15: \mathrm{mp}>230{ }^{\circ} \mathrm{C} ;{ }^{1} \mathrm{H} \mathrm{NMR}$ at $\mathrm{rt}$ $\left(\mathrm{CDCl}_{3}, 300 \mathrm{MHz}\right) \delta$ (ppm) 7.10 (br s, $\left.1 \mathrm{H}\right), 7.03$ (br s, 3H), 6.76 $(\mathrm{d}, 1 \mathrm{H}, J=2.1), 6.50(\mathrm{~d}, 1 \mathrm{H}, J=2.1) ;{ }^{19} \mathrm{~F}$ NMR at $243 \mathrm{~K}\left(d_{6}\right.$-acetone, $282.4 \mathrm{MHz}$ ), $\delta-65.133(\mathrm{~d}, J=48.9,2 \mathrm{H}, \mathrm{o}-\mathrm{F}),$, $(\mathrm{t}, J=24.6,1 \mathrm{~F}, \mathrm{p}-\mathrm{F}),-86.402(\mathrm{tt}, J=22.5$, tt, $J=5.7,2 \mathrm{~F}, \mathrm{~m}-\mathrm{F}$ ); TOF ES+ $311 \mathrm{~m} / z\left([\mathrm{M}-\mathrm{Br}]^{+}\right) ; \lambda_{\max }\left(\mathrm{CH}_{2} \mathrm{Cl}_{2}\right) / \mathrm{nm} \mathrm{314,} \mathrm{476.} 16$ was prepared by DDQ oxidation of $\mathbf{2}$ in the presence of $\mathrm{HCl}$. Spectral data for 16: $\mathrm{mp}>230{ }^{\circ} \mathrm{C} ; \lambda_{\max }\left(\mathrm{CH}_{2} \mathrm{Cl}_{2}\right) / \mathrm{nm} 470 ;{ }^{1} \mathrm{H}$ NMR at $333 \mathrm{~K}\left(d_{6}\right.$-acetone, $\left.300 \mathrm{MHz}\right) \delta 8.36(\mathrm{~d}, J=8.8,2 \mathrm{H})$, 7.77 (s, 2H), 7.76 (d, $J=8.7,2 \mathrm{H}), 6.50$ (d, $J=4.1,2 \mathrm{H}), 6.42$

Table 1. Selected crystallographic data of 3-6 and 8-9.

\begin{tabular}{|c|c|c|c|c|c|c|}
\hline & 3 & $4^{29}$ & 5 & 6 & 8 & 9 \\
\hline \multicolumn{7}{|c|}{ A. Crystal Data } \\
\hline $\begin{array}{l}\text { Formula } \\
\mathrm{Mw}\end{array}$ & $\begin{array}{c}\mathrm{C}_{15} \mathrm{H}_{7} \mathrm{~N}_{2} \mathrm{~F}_{5} \\
310.23\end{array}$ & $\begin{array}{c}\mathrm{C}_{15} \mathrm{H}_{11} \mathrm{~N}_{3} \mathrm{O}_{2} \\
265.27\end{array}$ & $\begin{array}{c}\mathrm{C}_{30} \mathrm{H}_{12} \mathrm{~F}_{10} \mathrm{~N}_{4} \mathrm{Zn} \\
683.81\end{array}$ & $\begin{array}{c}\mathrm{C}_{30} \mathrm{H}_{12} \mathrm{~F}_{10} \mathrm{~N}_{4} \mathrm{Cu} \\
681.98\end{array}$ & $\begin{array}{c}\mathrm{C}_{45} \mathrm{H}_{18} \mathrm{~N}_{6} \mathrm{~F}_{15} \mathrm{Mn} \\
982.59\end{array}$ & $\begin{array}{c}\mathrm{C}_{45} \mathrm{H}_{18} \mathrm{~N}_{6} \mathrm{~F}_{15} \mathrm{Fe} \\
983.50\end{array}$ \\
\hline Cryst. Color, habit & dark, needle & red, prism & red, plate & red, plate & $\begin{array}{l}\text { shiny green, } \\
\text { irregular }\end{array}$ & Red, platelet \\
\hline $\begin{array}{l}\text { Cryst. dimensions } \\
\text { (mm) }\end{array}$ & $0.40 \times 0.20 \times 0.20$ & $0.35 \times 0.25 \times 0.12$ & $0.25 \times 0.13 \times 0.05$ & $0.30 \times 0.15 \times 0.03$ & $0.15 \times 0.10 \times 0.10$ & $0.25 \times 0.12 \times 0.03$ \\
\hline $\begin{array}{l}\text { Cryst. System, } \\
\text { Lattice type }\end{array}$ & Orthorhombic & Orthorhombic & Monoclinic & Monoclinic & Monoclinic & Monoclinic \\
\hline Space group & $P 2{ }_{1}{ }_{1} 2_{1}(\# 19)$ & Pbca (\# 61) & $C 2 / c(\# 15)$ & $P 2_{1} / c(\# 14)$ & $P 2_{1} / n(\# 14)$ & $P 2_{1} / n(\# 14)$ \\
\hline$a(\AA)$ & $8.9173(6)$ & $14.6809(16)$ & $55.915(9)$ & $15.450(2)$ & $10.3595(5)$ & $10.2500(15)$ \\
\hline$b(\AA)$ & $9.1568(6)$ & $7.4146(9)$ & $8.176(2)$ & $9.1389(9)$ & $13.5804(9)$ & 13.6111(19) \\
\hline$c(\AA)$ & $15.3482(10)$ & $24.350(3)$ & $12.195(2)$ & $19.725(2)$ & $26.6890(19)$ & $27.829(4)$ \\
\hline (deg) & 90 & 90 & $92.900(10)$ & $112.500(10)$ & $94.098(3)$ & $94.546(4)$ \\
\hline$V\left(\AA^{3}\right)$ & $1253.24(14)$ & $2650.6(6)$ & $5567.9(19)$ & $2573.1(5)$ & $3745.2(4)$ & $3870.3(10)$ \\
\hline$Z$ & 4 & 8 & 8 & 4 & 4 & 4 \\
\hline$D_{\mathrm{c}}\left(\mathrm{g} / \mathrm{cm}^{3}\right)$ & 1.644 & 1.329 & 1.631 & 1.760 & 1.743 & 1.688 \\
\hline$F_{000}$ & 624.00 & 1104.00 & 2720.00 & 1356.00 & 1960.00 & 1964.00 \\
\hline$\mu(\operatorname{Mo~K} \alpha)\left(\mathrm{mm}^{-1}\right)$ & 0.151 & 0.092 & 0.978 & 0.952 & 0.474 & 0.507 \\
\hline \multicolumn{7}{|c|}{ B. Intensity Measurements } \\
\hline Diffractometer & $\begin{array}{l}\text { Rigaku/ADSC } \\
\text { CCD }\end{array}$ & Bruker X8 APEX & $\begin{array}{l}\text { Rigaku/ADSC } \\
\text { CCD }\end{array}$ & $\begin{array}{c}\text { Rigaku/ADSC } \\
\text { CCD }\end{array}$ & Bruker X8 APEX & $\begin{array}{l}\text { Rigaku/ADSC } \\
\text { CCD }\end{array}$ \\
\hline $\begin{array}{l}\text { Total No. of refl. } \\
\text { measured }\end{array}$ & $\begin{array}{c}11430 \\
\left(\mathrm{R}_{\mathrm{int}}=0.053\right)\end{array}$ & $\begin{array}{c}54647 \\
\left(\mathrm{R}_{\mathrm{int}}=0.0325\right)\end{array}$ & $\begin{array}{c}22844 \\
\left(\mathrm{R}_{\mathrm{int}}=0.061\right)\end{array}$ & $\begin{array}{c}23088 \\
\left(\mathrm{R}_{\mathrm{int}}=0.0814\right)\end{array}$ & $\begin{array}{c}65695 \\
\left(\mathrm{R}_{\mathrm{int}}=0.0646\right)\end{array}$ & $\begin{array}{c}29858 \\
\left(\mathrm{R}_{\text {int }}=0.124\right)\end{array}$ \\
\hline Corrections & $0.769 \sim 0.970$ & $0.941 \sim 0.989$ & $0.550 \sim 0.952$ & $0.690 \sim 0.972$ & $0.0815 \sim 0.954$ & $0.815 \sim 0.985$ \\
\hline \multicolumn{7}{|c|}{ C. Structure Solution and Refinement } \\
\hline Structure solution & $\begin{array}{l}\text { Direct methods } \\
\quad \text { (SIR92) }\end{array}$ & $\begin{array}{l}\text { Direct methods } \\
\quad \text { (SIR92) }\end{array}$ & $\begin{array}{l}\text { Direct methods } \\
\quad \text { (SIR92) }\end{array}$ & $\begin{array}{l}\text { Direct methods } \\
\quad \text { (SIR92) }\end{array}$ & $\begin{array}{l}\text { Direct methods } \\
\quad \text { (SIR92) }\end{array}$ & $\begin{array}{l}\text { Patterson methods } \\
\text { (DIRDIF92 PATTY) }\end{array}$ \\
\hline $\begin{array}{l}\text { No. observation } \\
(I>0.00 \sigma(I))\end{array}$ & 2696 & 3122 & 6020 & 5293 & 7314 & 6779 \\
\hline No. variables & 203 & 185 & 407 & 406 & 604 & 605 \\
\hline Refl./para. ratio & 13.28 & 16.88 & 14.79 & 13.04 & 12.11 & 11.20 \\
\hline$R 1 ; w R 2$ & $0.0407 ; 0.0622$ & $0.0621 ; 0.1160$ & $0.1374 ; 0.2200$ & $0.1067 ; 0.1359$ & $0.0587 ; 0.1076$ & $0.1273 ; 0.1670$ \\
\hline$R 1 ; w R 2$ & $0.0292 ; 0.0588$ & $0.0403 ; 0.1051$ & $0.0890 ; 0.1855$ & $0.0556 ; 0.1203$ & $0.0388 ; 0.1006$ & $0.0607 ; 0.1238$ \\
\hline GOF & 0.904 & 1.074 & 0.883 & 0.964 & 1.080 & 0.830 \\
\hline
\end{tabular}


$\left(\mathrm{dd}, J=4.4,{ }^{\mathrm{dd}} J=1.5,2 \mathrm{H}\right)$; HR ESI $(+)$ calcd. $266.0930 \mathrm{~m} / \mathrm{z}$ (for $\mathrm{C}_{15} \mathrm{H}_{12} \mathrm{~N}_{3} \mathrm{O}_{2}$ ), found $266.09028 \mathrm{~m} / z$, (-) calcd. 226.9415 $\mathrm{m} / z$ (for $\mathrm{C}_{8} \mathrm{HN}_{2} \mathrm{O}_{2}{ }^{35} \mathrm{Cl}_{2}$ ), found 226.9423 .

STM determination of the self-assemblies 11 and 13-14. For adsorption of the self-assemblies 11 and 13-14 onto a graphite surface (MikroMasch, grade ZYA), the compound was first dissolved in methanol at a concentration of $c a .1 .0 \times 10^{-5} \mathrm{M}$. A drop of the solution was placed onto a freshly cleaved surface and left to dry in air either at room temperature or at $100{ }^{\circ} \mathrm{C}$ for several hours. The drops spread evenly over the surface, resulting in uniform coverage of the entire surface with each compound. STM topographical images were obtained using a scanning tunneling microscope (SOLVER P-47, NT-MDT) under ambient conditions. STM tips were mechanically cut from a 0.25 $\mathrm{mm} \mathrm{Pt/Ir} \mathrm{wire} \mathrm{and} \mathrm{tunneling} \mathrm{currents} \mathrm{between} 30$ and $60 \mathrm{pA}$ were employed. The sample bias voltage and the scan frequency were 100 to $200 \mathrm{mV}$ and between 0.5 and $3 \mathrm{~Hz}$, respectively. Constant-current mode was employed to investigate the static properties of the structures. Multiple-tip effects, which can pro- duce misleading features in STM images, were removed by a repeated tip sharpening process in situ at a graphite step edge. All of the images shown are raw data with mean plane subtraction to reduce the thermal drift effect.

\section{Results and Discussion}

Dipyrrins become more stable when substituted with electron-withdrawing groups and more conformationally mobile when unsubstituted in the $\beta$-positions such as with compounds $\mathbf{3}$ and 4. Dipyrromethanes $\mathbf{1}$ and $\mathbf{2}$ were prepared following the literature method from free pyrrole and perfluorobenzaldehyde (or 4-nitrobenzaldehyde for 2) and oxidized using 2,3-dichloro5,6-dicyano-1,4-benzoquinone (DDQ) to obtain 3 and 4. Metal complexes 5-9 were simply prepared by treatment of a $\mathrm{CH}_{3} \mathrm{CN}$ solution of dipyrrin 3 with MeOH solutions of $\mathrm{Zn}^{2+}, \mathrm{Cu}^{2+}, \mathrm{Ni}^{2+}$, $\mathrm{Mn}^{3+}$ and $\mathrm{Fe}^{3+}$ chloride salts. Single crystals of 3-6 and 8-9 were grown by the vapor diffusion of hexane into $\mathrm{CH}_{2} \mathrm{Cl}_{2}$ solutions and each structure was defined by X-ray diffraction analysis.

Table 2. Selected crystallographic data of 10-13 and 15-16.

\begin{tabular}{|c|c|c|c|c|c|}
\hline & $10^{28}$ & $11^{28}$ & 12 & $15^{28}$ & $16^{29}$ \\
\hline \multicolumn{6}{|c|}{ A. Crystal Data } \\
\hline Formula & $\mathrm{C}_{30} \mathrm{H}_{16} \mathrm{Cl}_{4} \mathrm{ZnN}_{4} \mathrm{~F}_{10}$ & $\mathrm{C}_{30} \mathrm{H}_{16} \mathrm{~N}_{4} \mathrm{~F}_{10} \mathrm{ZnBr}_{4} \cdot \mathrm{C}_{3} \mathrm{H}_{6} \mathrm{O}$ & $\mathrm{C}_{30} \mathrm{H}_{16} \mathrm{Cl}_{4} \mathrm{CuN}_{4} \mathrm{~F}_{10}$ & $\mathrm{C}_{15} \mathrm{H}_{8} \mathrm{~N}_{2} \mathrm{~F}_{5} \mathrm{Br}$ & $\mathrm{C}_{23} \mathrm{H}_{13} \mathrm{~N}_{5} \mathrm{O}_{4} \mathrm{Cl}_{2}$ \\
\hline Mw & 829.66 & 1065.54 & 827.81 & 391.14 & 494.28 \\
\hline Cryst. Color, habit & red, platelet & blue, block & dark, irregular & red, chip & red, plate \\
\hline $\begin{array}{l}\text { Cryst. dimensions } \\
\qquad(\mathrm{mm})\end{array}$ & $0.50 \times 0.30 \times 0.08$ & $0.50 \times 0.20 \times 0.20$ & $0.30 \times 0.20 \times 0.12$ & $0.50 \times 0.25 \times 0.13$ & $0.35 \times 0.10 \times 0.03$ \\
\hline $\begin{array}{l}\text { Cryst. System, } \\
\text { Lattice type }\end{array}$ & $\begin{array}{l}\text { Orthorhombic, } \\
\text { primitive }\end{array}$ & $\begin{array}{l}\text { Monoclinic, } \\
\text { primitive }\end{array}$ & $\begin{array}{l}\text { Orthorhombic, } \\
\text { primitive }\end{array}$ & Triclinic, & $\begin{array}{l}\text { Monoclinic, } \\
\text { primitive }\end{array}$ \\
\hline Space group & $\operatorname{Pbcn}(\# 60)$ & $\mathrm{P} 2{ }_{1} / \mathrm{n}(\# 14)$ & Pbcn (\# 60) & $\mathrm{P}_{\overline{1}}^{-}(\# 2)$ & $\mathrm{P} 2{ }_{1} / \mathrm{n}(\# 14)$ \\
\hline$a(\AA)$ & $14.6458(6)$ & $10.4590(7)$ & 14.818 & $8.2509(2)$ & $10.508(2)$ \\
\hline$b(\AA)$ & $11.9140(6)$ & $18.2100(9)$ & 11.405 & $10.9918(1)$ & $19.587(3)$ \\
\hline$c(\AA)$ & $18.0743(9)$ & $20.058(1)$ & 18.408 & $17.5200(6)$ & $11.039(2)$ \\
\hline$(\operatorname{deg})$ & 90 & $105.072(2)$ & 90 & $79.310(9)^{*}$ & $107.970(10)$ \\
\hline$Z$ & 4 & 4 & 4 & 4 & 4 \\
\hline$D_{\mathrm{c}}\left(\mathrm{g} / \mathrm{cm}^{3}\right)$ & 1.747 & 1.919 & 1.767 & 1.754 & 1.519 \\
\hline$F_{000}$ & 1648.00 & 2064.00 & 1644.00 & 768.00 & 1008.00 \\
\hline$\mu(\operatorname{MoK} \alpha)\left(\mathrm{cm}^{-1}\right)$ & 1.207 & 5.097 & 1.137 & 2.838 & 0.344 \\
\hline \multicolumn{6}{|c|}{ B. Intensity Measurements } \\
\hline Diffractometer & Rigaku / ADSC CCD & Bruker X8 APEX & Bruker X8 APEX & Rigaku / ADSC CCD & Bruker X8 APEX \\
\hline $\begin{array}{c}\text { Total No. of refl. } \\
\text { measured }\end{array}$ & $\begin{array}{c}28992 \\
\left(\mathrm{R}_{\mathrm{int}}=0.05940\right)\end{array}$ & $\begin{array}{c}33094 \\
\left(R_{\text {int }}=0.05739\right)\end{array}$ & $\begin{array}{c}32908 \\
\left(\mathrm{R}_{\mathrm{int}}=0.0372\right)\end{array}$ & $\begin{array}{c}13327 \\
\left(\mathrm{R}_{\mathrm{int}}=0.04372\right)\end{array}$ & $\begin{array}{c}34997 \\
\left(\mathrm{R}_{\mathrm{int}}=0.0710\right)\end{array}$ \\
\hline Corrections & $0.7515 \sim 1.0000$ & $0.6906 \sim 1.0000$ & $0.471 \sim 0.711$ & $0.7733 \sim 1.0000$ & $0.756 \sim 0.990$ \\
\hline \multicolumn{6}{|c|}{ C. Structure Solution and Refinement } \\
\hline Structure solution & $\begin{array}{l}\text { Patterson methods } \\
\text { (DIRDIF92 PATTY) }\end{array}$ & $\begin{array}{l}\text { Direct methods } \\
\quad \text { (SIR 97) }\end{array}$ & $\begin{array}{l}\text { Direct methods } \\
\quad \text { (SIR 92) }\end{array}$ & $\begin{array}{l}\text { Direct methods } \\
\quad \text { (SIR 97) }\end{array}$ & $\begin{array}{l}\text { Direct methods } \\
\quad \text { (SIR 92) }\end{array}$ \\
\hline No. variables & 230 & 494 & 230 & 427 & 319 \\
\hline Refl./para. ratio & 15.11 & 16.64 & 16.15 & 14.22 & 12.04 \\
\hline$R 1 ; w R 2$ & $0.0486 ; 0.0700$ & $0.0585 ; 0.0963$ & $0.0560 ; 0.0801$ & $0.0799 ; 0.1493$ & $0.0756 ; 0.1290$ \\
\hline$R 1 ; w R 2$ & $0.027 ; 0.0700$ & $0.031 ; 0.035$ & $0.0310 ; 0.0701$ & $0.043 ; 0.059$ & $0.0456 ; 0.1087$ \\
\hline GOF & 0.926 & 0.842 & 1.047 & 1.131 & 1.09 \\
\hline
\end{tabular}


(a)

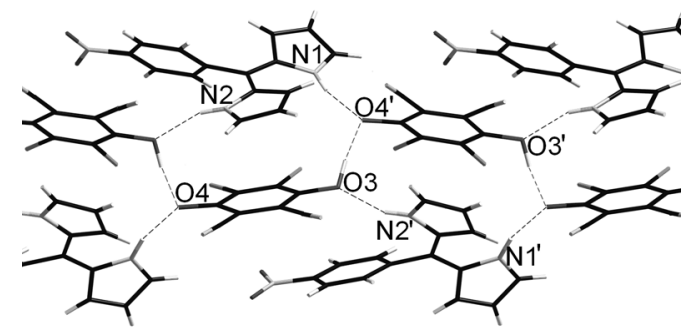

(b)
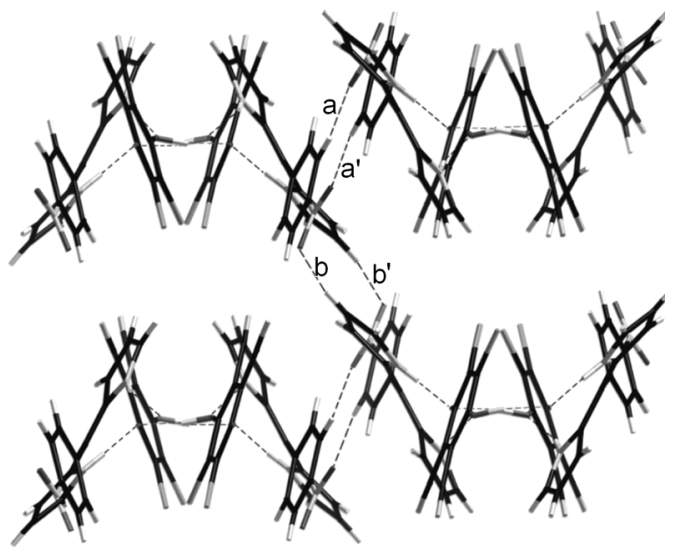

Figure 18. 3D packing diagrams of 16; [(a) top view and (b) side view] showing the hydrogen-bonding system between protonated dipyrromethene and deprotonated/reduced DDQ; hydrogen-bonds are denoted by dotted red $(\mathrm{OH} \cdots \mathrm{O})$, blue $(\mathrm{NH} \cdots \mathrm{O})$, and black $(\mathrm{CH} \cdots \mathrm{O})$ lines.
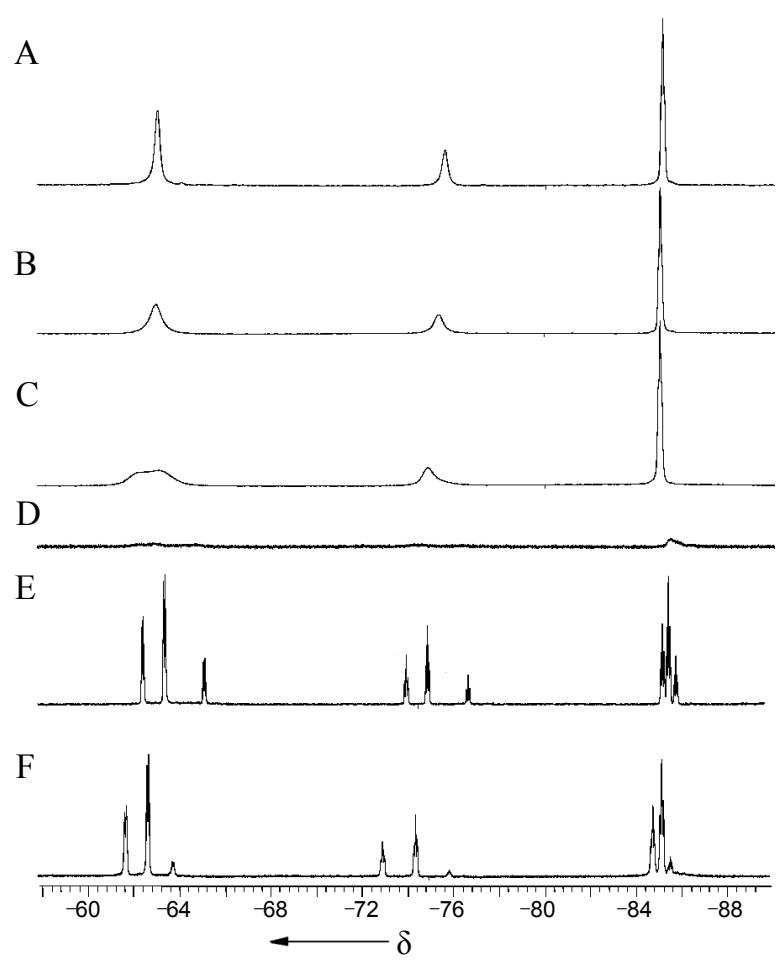

Figure 19. Variable temperature ${ }^{19} \mathrm{~F}$ NMR spectra of $\mathbf{1 1}$ in $d_{6}$-DMSO (A-C) and $d_{6}$-acetone (D-F); (A) $353 \mathrm{~K}$, (B) $333 \mathrm{~K}$, (C) $300 \mathrm{~K}$, (D) $300 \mathrm{~K}$, (E) $253 \mathrm{~K}$, and (F) $223 \mathrm{~K}$.

As shown in Figures 3 and 4, the $\mathrm{ZnD}_{2} 5$ and $\mathrm{CuD}_{2} 6$ have tetrahedral structures while $\mathrm{MnD}_{3} 8$ (Figure $4 \mathrm{a}$ ) and $\mathrm{FeD}_{3} 9$ (Figure $4 \mathrm{~b})$ have octahedral coordinations. $\mathrm{ZnD}_{2} 5$ shows a $c a .45^{\circ}$ dihe-

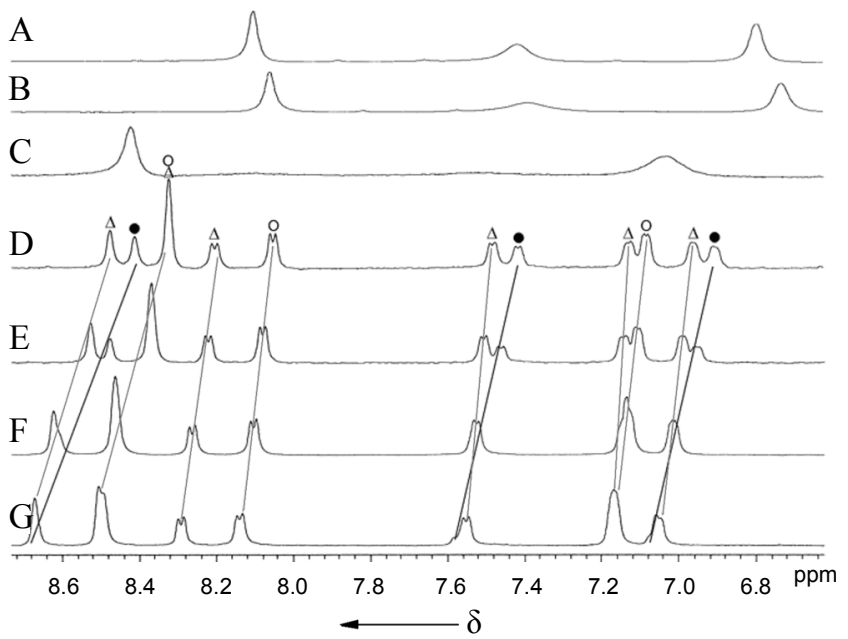

Figure 20. Various temperature $1 \mathrm{H}$ NMR spectra of $\mathbf{1 1}$ in $d_{6}$-DMSO (A-B) and $d_{6}$-acetone $(\mathrm{C}-\mathrm{G})$; (A) $353 \mathrm{~K}$, (B) $333 \mathrm{~K}$, (C) $300 \mathrm{~K}$, (D) $253 \mathrm{~K}$, (E) $223 \mathrm{~K}$, (F) $203 \mathrm{~K}$, and (G) 183; o conformation I, • conformation II, and $\boldsymbol{\Delta}$ conformation III.

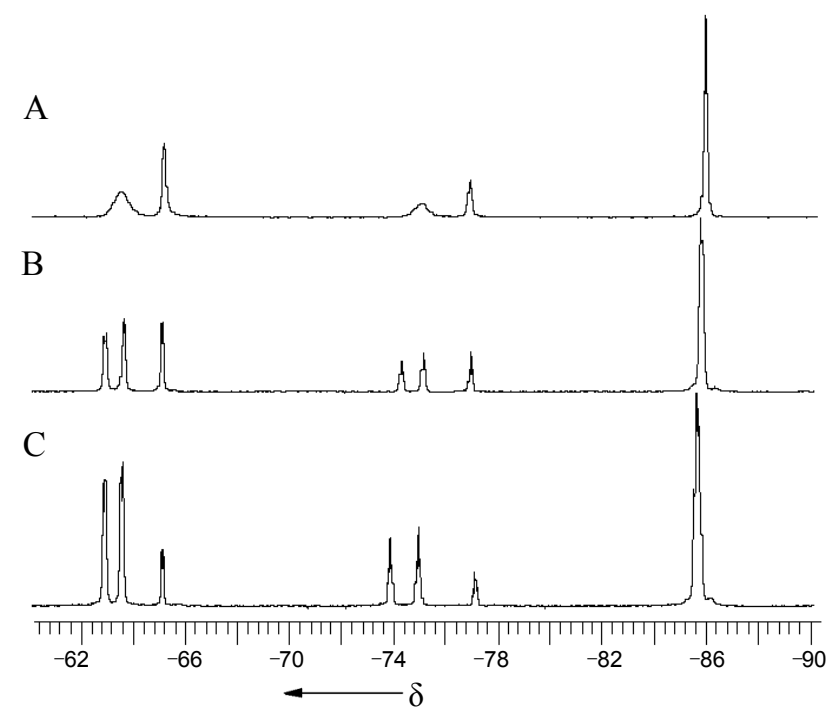

Figure 21. Variable temperature ${ }^{19} \mathrm{~F}$ NMR spectra of $\mathbf{1 5}$ in $d_{6}$-acetone; (A) $300 \mathrm{~K}$, (B) $260 \mathrm{~K}$, and (C) $220 \mathrm{~K}$.

dral angle for the planes along the two chelating dipyrrin ligands and the same angle is $60^{\circ}$ for $\mathrm{CuD}_{2} 6$. As shown in the selected crystallographic data in Table 1, all the single crystals of the metal complexes have monoclinic systems. The NMR spectra of the metal complexes 6-9 exhibit signals over a large chemicalshift range or completely disappeared due to the paramagnetism of the metal centers while the diamagnetic $\mathrm{Zn}$ complexes 5 shows typical chemical shifts in their NMR spectrum. As reported in previously, ${ }^{31-33}$ the optical spectra of the metal complexes showed strong absorption bands newly appearing along with metal complexation. Similarly, the optical spectrum of the trivalent metal complexes $\mathbf{8}$ and $\mathbf{9}$ are also dramatically changed from that of the ligand. Relatively numerous splittings of molecular orbitals by increasing the ligation number caused the absorption bands to be slightly broadened and weaker compared to the divalent metal complexes. 

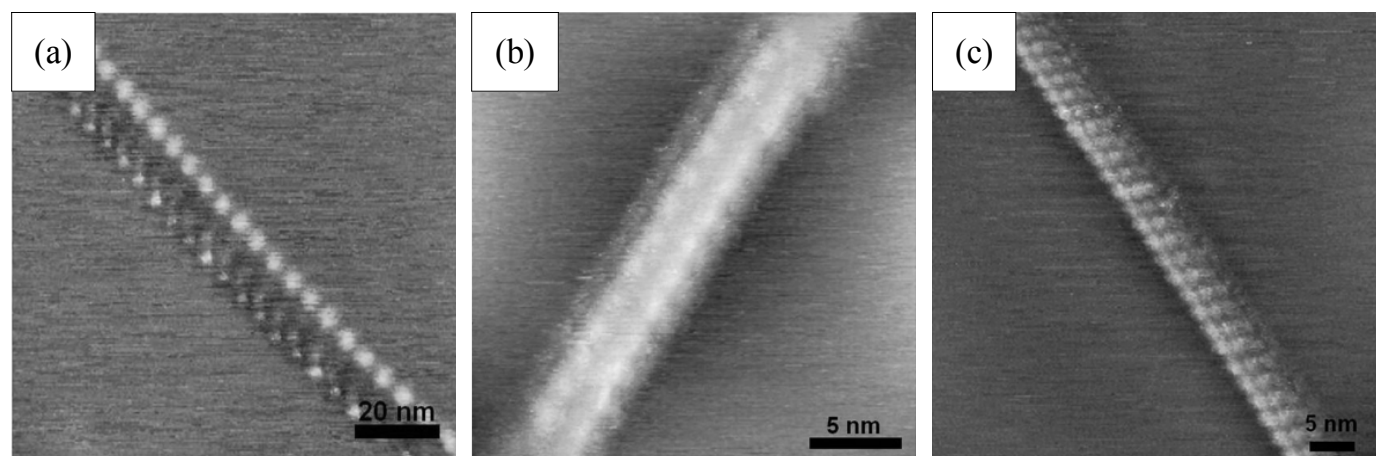

Figure 22. STM patterns for 11 (a), 13 (b), and 14 (c).

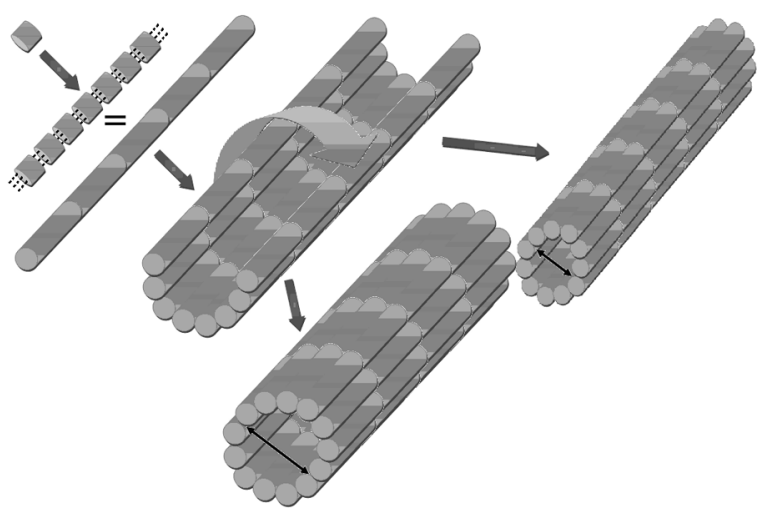

Figure 23. Graphic displays for the explanation of tubular formations.

As shown in Figure 1, dipyrrin can exhibit three possible conformations. Conformation I is observed in the general crystal structure of free-based dipyrrins. As shown in Figure 2, the crystal structures of $\mathbf{3}$ and $\mathbf{4}$ show the stable type I conformation formed via intramolecular hydrogen-bonding between imino-N and amino-NH. Similarly, general metal complexes of dipyrrin ligands takes conformation I by clamping both the imino- and amino-N atoms by the central metals (Figures 3 and 4). The selfassemblies containing protonated dipyrrins, $\mathrm{ZnCl}_{4}(\mathrm{HD})_{2} \mathbf{1 0}$, $\mathrm{ZnBr}_{4}(\mathrm{HD})_{2} \mathbf{1 1}, \mathrm{CuCl}_{4}(\mathrm{HD})_{2} \mathbf{1 2}, \mathrm{CuBr}_{4}(\mathrm{HD})_{2} \mathbf{1 3}, \mathrm{NiBr}_{4}(\mathrm{HD})_{2}$ 14, and $\mathrm{HDBr} 15$ were formed when hydrogen halides $(\mathrm{HCl}$ for 10 and 12, $\mathrm{HBr}$ for 11 and 13-15) were added to $\mathrm{CH}_{3} \mathrm{CN}$ solutions of 5-9. Each single crystal was obtained by slow evaporation of a solution in water and methanol and the structures of the self-assemblies 10-12 and 15 were defined by X-ray diffraction analysis. The single crystals are hygroscopic and readily become viscous and amorphous and dark color.

As shown in Figures 5, 8, 11, and 15, the initial conformation of the dipyrrin components were converted to the type II conformation. Introduction of halide anions caused the cleavage of the original metal-N bonds and led to formation of new metalhalide bonds. The metal tetrahalide $\left(\mathrm{ML}_{4}\right)$ complexes were the first species formed within this motif. Additionally, the dipyrrin molecules were protonated during the treatment with hydrogen halide (HL). The ML4s are dianions and the resulting self-assemblies have a net charge of zero when generated with two protonated-dipyrrins (HDs), where the positive charges are delo- calized throughout the entire aromatic dipyrrin. Similarly, the negative charge is also delocalized through the $\mathrm{ML}_{4}$ units. The four halide arms of $\mathrm{ML}_{4} \mathrm{~s}$ which can generate four different hydrogen-bonds, link with four dipyrrin components (two protonated NHs and two original amino-NHs). As a result, the superstructure assembly generated through hydrogen-bonding interactions has zero net-charge. These co-crystals also exhibit rather unique multi-dimensional arrangements. As shown in Figures 6,9 and 13, the three-dimensional packing diagrams display complicated but well-repeated patterns. Within the continuously repeating hydrogen-bonding network, the two-dimensionally aligned molecules are tightly linked with each other and the final structures occur as two dimensional layered superstructures. The side views of the alignments help in recognizing the individual superstructural layers (Figures 7, 10 and 14, especially the individual super-structural layer denoted in Figure 7 (c), as an example). The molecular arrangements in the selfassemblies are very similar, but the distances between each layer are due to the differences in the van der Waals bond distances and the dihedral angle of the $\mathrm{ML}_{4}$ tetrahedral units. $\mathrm{CuCl}_{4} \mathrm{com}$ ponents in the crystal exhibited a more distorted tetrahedral coordination than $\mathrm{ZnBr}_{4}$ (Figure 12).

Self-assembly (Figure 15) of HD and a bromide anion shows ribbon-like molecular arrangements as a result of the hydrogenbonding networks. Interestingly, it is a racemic mixture having two different helical structures as denoted with black arrows in Figure 15. As mentioned above, the type I and II conformations were well represented in the various solid states. On the other hand, the type III conformation (Figure 1) was as yet unknown until the crystal structure of compound 16 (Figure 17) was determined.

The single crystal of $\mathbf{1 6}$ was obtained by evaporation of a solution of methanol and trace $\mathrm{HCl}$ in water. The crystal structure as presented in Figures 17 and 18(a) contains a dipyrrin component in an asymmetric type III conformation. Similar to the previous self-assemblies, the dipyrrin units in the crystal were protonated. A crystal of HDs and deprotonated diquinolate components was formed in the presence of acid. The structure of the self-assembly was tightened by three different types of hydrogen-bonds, $\mathrm{O}-\mathrm{H} \cdots \mathrm{O}(1.53 \AA), \mathrm{N}-\mathrm{H} \cdots \mathrm{O}(1.85$ and $2.01 \AA)$ and $\mathrm{C}-\mathrm{H} \cdots \mathrm{O}$ $(2.59$ and $2.71 \AA){ }^{29}$

As shown in the crystal structures above, the conformational differences of the three dipyrrins were confirmed in the solid 
state. In order to examine conformational changes in solution NMR spectral analysis at various temperatures was utilized. Figure 19 shows the various temperature ${ }^{19} \mathrm{~F}$ NMR spectra of 11. At higher temperatures, the spectrum showed the presence of one conformer. As the temperature was lowered, the peaks progressively broadened and split. As shown in spectrum D ( $d_{6}$-acetone solution), the F-peaks almost disappeared at room temperature. With continued lowering of the temperature, the F-signals reappeared showing all three different conformation types. One can confidently distinguish each set of peaks of the three conformations from the temperature dependence of the ${ }^{1} \mathrm{H}$ NMR spectra of $\mathbf{1 1}$ (Figure 20). Similarly, ${ }^{19}$ F NMR spectra of 15 were considered as a three conformational mixture (Figure 21).

Further investigation of the arrangements of these self-assemblies in both the solid and solution states continued with microscopic analysis. Drops of $\mathrm{MeOH}$ solutions of compounds 11 and 13-14 were placed on highly-oriented pyrolytic graphite surfaces (HOPGS) and the solvent was allowed to completely evaporate. The graphite plates were connected to a low-current preamplifier electrode for STM-analysis. The STM images are presented in Figure 22. As shown in Figure 23, the self-assemblies on the graphite surface initially formed one-dimensional arrangements via the specific interactions of the components, which might be considered as hydrogen-bonds as described above. The 1D-aligned assembly continuously interacts with another 1D-aligned unit to form tubular bunches. These tubular structures might well be in accordance with the supermolecular layered structures of the crystals reported above. The STM image in Figure 22(a) likely represents a case where the alignment was stopped before completing the tubular arrangement.

\section{Conclusion}

Three possible conformations of dipyrrins, which are shown as type I, II and III in Figure 1, have been observed in both the solid and solution states. The crystals of free-base dipyrrin and metal-dipyrrin complexes reveal type I conformation. Alternately, self-assemblies built via metal-N bond cleavage followed by new coordinate-bond generation to produce metal tetrahalides, protonation of imino-nitrogen, and hydrogen-bond formation between halide atoms and $\mathrm{NH}$ groups showed type II conformation. The co-crystalline structure of self-assembly 16 provided the last conformation, type III. Additionally, the three conformations were observed in solution using variable-temperature NMR. The layered arrangements of the self-assemblies generated tubular bunches on a HOPGS as shown via STM.

Studies on alternative dimensional arrangements using selfassemblies built by diverse combinations of flexible components are ongoing.

Acknowledgments. This work was supported by the Natural Sciences and Engineering Research Council (NSERC) of Canada. SBS and JRH thank the KOSEF funded by the MOST
(R11-2007-012-02001-0 and R01-2007-000-20237-0).

\section{References}

1. Desiragu, G. R. Crystal Engineering: The Design of Organic Solids; Elsevier: Amsterdam, 1989.

2. Lehn, J. M. Supramolecular Chemistry: Concepts and Perspectives; VCH: Weinheim, 1995.

3. Comprehensive Supramolecular Chemistry; Atwood, J. L., Davies, J. E. D., MacNicol, D. D., Vogtle, F., Eds.; Pergamon: Oxford, 1996.

4. Desiraju, G. R. Nature 2001, 412, 397.

5. Hollingsworth, M. D. Science 2002, 295, 2410.

6. MacDonald, J. C.; Dorrestein, P. C.; Pilley, M. M.; Foote, M. M.; Lundburg, J. L.; Denning, R. W.; Schultz, A. J.; Manson, J. L. J. Am. Chem. Soc. 2000, 122, 11692.

7. Moulton, B.; Zaworotko, M. J. Chem. Rev. 2001, 101, 1629.

8. Sommerdijk, N. A. J. M. Angew. Chem. Int. Ed. 2003, 42, 3572.

9. Daisuke, U.; Yusuke, U.; Takashi, O. Science 2009, 326, 120-123.

10. Yaghi, O. M.; O'Keeffe, M.; Ockwig, N. W.; Chae, H. K.; Eddaudi, M.; Kim, J. Nature 2003, 423, 705-714.

11. Ko, Y. H.; Kim, E.; Hwang, I.; Kim, K. Chem. Commun. 2007, 1305-1315.

12. Sharma, C. V. K.; Broker, G. A.; Huddleston, J. G.; Baldwin, J. W.; Metzger, R. M.; Rogers, R. D. J. Am. Chem. Soc. 1999, 121, 1137.

13. Yanagi, H.; Mukai, H.; Ikuta, K.; Shibutani, T.; Kamikado, T.; Yokoyama, S.; Mashiko, S. Nano Lett. 2002, 2, 601.

14. Chen, Q.; Dolphin, D. Can. J. Chem. 2002, 80, 1668.

15. Thompson, A.; Rettig, S. J.; Dolphin, D. Chem. Commun. 1999, 631.

16. Zhang, Y.; Thompson, A.; Rettig, S. J.; Dolphin, D. J. Am. Chem. Soc. 1998, 120, 13537.

17. Maeda, H.; Mihashi, Y.; Haketa, Y. Org. Lett. 2008, 10, 3179.

18. Maeda, H. Eur. J. Org. Chem. 2007, 5313.

19. Sessler, J. L.; Berthon-Gelloz, G.; Gale, P. A.; Camiolo, S.; Anslyn, E. V.; Anzenbacher, P., Jr.; Furuta, H.; Kirkovits, G. J.; Lynch, V. M.; Maeda, H.; Morosini, P.; Scherer, M.; Shriver, J.; Zimmerman, R. S. Polyhedron 2003, 22, 2963.

20. Sessler, J. L.; Jayawickramarajah, J.; Sherman, C. L.; Brodbelt, J. S. J. Am. Chem. Soc. 2004, 126, 11460.

21. Katayev, E. A.; Boev, N. V.; Khrustalev, V. N.; Ustynyuk, Y. A.; Tananaev, I. G.; Sessler, J. L. J. Org. Chem. 2007, 72, 2886.

22. Desiraju, G. R. Angew. Chem. Int. Ed. Engl. 1995, 34, 2311.

23. Desiraju, G. R. Chem. Commun. 1997, 1475.

24. Bond, A. D. CrystEngComm. 2007, 9, 833.

25. Simard, M.; Su, D.; Wuest, J. D. J. Am. Chem. Soc. 1991, 113, 4696.

26. Fournier, J. H.; Maris, T.; Wuest, J. D.; Guo, W. Z.; Galoppini, E. J. Am. Chem. Soc. 2003, 125, 1002.

27. Falk, H. In The Chemistry of Linear Oligopyrroles and Bile Pigments; Springer-Verlag Wien: New York, 1989; pp 108.

28. Shin, J. Y.; Dolphin, D.; Patrick, B. O. Cryst. Growth Des. 2004, 4, 659.

29. Shin, J. Y.; Patrick, B. O.; Dolphin, D. CrystEngComm. 2008, 10, 960.

30. Shin, J. Y.; Patrick, B. O.; Dolphin, D. Org. Biomol. Chem. 2009, 7, 2032

31. Brückner, C.; Karunaratne, V.; Rettig, S.; Dolphin, D. Can. J. Chem. 1996, 74, 2182.

32. Miao, Q.; Shin, J. Y.; Patrick, B. O.; Dolphin, D. Chem. Commun. 2009, 2541-2543.

33. Brückner, C.; Zhang, Y.; Rettig, S. J.; Dolphin, D. Inorg. Chim. Acta. 1997, 263, 279-286. 